\title{
On the direction of Coriolis force and the angular momentum conservation
}

\author{
Sobre a direção da força de Coriolis e da conservação do momento angular \\ Eduardo Herrera, Sigrid Morett \\ Universidad Nacional Autónoma de México, México, D.F
}

Recebido em 29 de janeiro de 2016. Revisado em 15 de março de 2016. Aceito em 19 de março de 2016

\begin{abstract}
This paper presents a qualitative analysis of the Coriolis force direction using the conservation of angular momentum and comparing it with quantitative calculation. In order to understand the implications of the theoretical expression for Coriolis force, we show the theory to students and teachers in a different way. When an object is on a rotating flat plate or rotating sphere (with constant angular velocity), has an associated tangential velocity due to rotation. If the object moves toward a larger (smaller) radius, in order to conserve angular momentum, there must be a force to reduce (increase) the tangential velocity. If the object moves parallel to tangential velocity, for angular momentum conservation, when tangential velocity increases (decreases) there must be a force which impels the object toward a larger (smaller) radius.
\end{abstract}

Keywords: Coriolis force, angular momentum, physics teaching.

Este trabalho apresenta uma análise qualitativa da direção da força de Coriolis usando a conservação do momento angular e comparando-a com cálculo quantitativo. A fim de compreender as implicações da expressão teórica para a força de Coriolis, se mostra a teoria de uma maneira distinta, para alunos e professores. Quando um objeto está sobre uma superficie plana ou uma esfera em rotaçao (com velocidade angular constante), possui uma velocidade tangencial devido à rotação. Se o objecto se move para um raio maior (menor), a fim de conservar o momento angular, deve haver uma força para reduzir (aumentar) a velocidade tangencial. Se o objeto se move paralelo à velocidade tangencial, por conservação do momento angular, quando aumenta (diminui) a velocidade tangencial (diminui) deve haver uma força impele o objeto para um raio maior (menor).

Palavras-chave: força de Coriolis, momento angular, ensino de física.

\section{Introduction}

More than 180 years have passed since Gaspard Gustav de Coriolis (1792-1843) published his works $[1,2]$, but their implications remain fresh and have been applied in many and different topics, from serious and scientific ones to informal and anecdotic ones.

Coriolis force is a classic subject studied in every Mechanics course in Bachelor of Physics or Engineering, when the aspects related to inertial and non-inertial systems are reviewed.

*Endereço de correspondência: herreraztegui@gmail.com
This subject is fundamental because of its connections with other important subjects from Physics e.g. rotational systems, angular momentum and its conservation, rigid body, etc., which are often only partially understood by students, studying them for the first time.

And a growing number of students in Earth Sciences related disciplines (Atmospheric Sciences, Oceanography, Hydrology, Space Physics, etc.) also need a good comprehension of this subject [3-5].

We want to show an alternative way to obtain the direction of Coriolis force qualitatively by using the angular momentum conservation, as this is an intuitive help for students in understanding this 
force. We want to help students develop a physics based intuition in order to predict the direction of the Coriolis force, in a way which makes more sense than the mathematical conclusion "it goes to the right side of the movement on the north hemisphere". Mathematics of course describes it, but it does not explain why the force operates this way. Schmidt [6] did an approach to this topic, but in that work the angular momentum conservation explanation was not shown explicitly and the author used other concepts like centripetal force for it.

We briefly present the known theory of Coriolis force and angular momentum conservation in sections 2 and 2.1. Then, by only using angular momentum conservation we theoretically obtain the direction which the force should have (qualitatively and quantitatively) in two and three dimensions (sections 3.1 and 3.2 respectively), which is the substantial section of this paper. Finally, in section 4, the conclusions are presented.

\section{The known theory}

A vector $\mathbf{A}$ (vectors are denoted by bold) has a time change rate in the inertial reference system (subscript $i$ ) [7, 8],

$$
\left(\frac{d \mathbf{A}}{d t}\right)_{i}=\frac{d \mathbf{A}}{d t}+\mathbf{\Omega} \times \mathbf{A}
$$

where $d \mathbf{A} / d t$ is the time change rate in the rotating reference frame (non-inertial) and $\boldsymbol{\Omega}$ is the angular velocity vector.

Applying this to the change of the position vector $\mathbf{r}(x, y, z)$ of a material point in the Cartesian coordinate system, we obtain

$$
\left(\frac{d \mathbf{r}}{d t}\right)_{i}=\frac{d \mathbf{r}}{d t}+\mathbf{\Omega} \times \mathbf{r}
$$

And defining velocity vector as $\mathbf{V}=d \boldsymbol{r} / d t=$ $(d x / d t, d y / d t, d z / d \mathrm{t})=(u, v, w)$, the Eq. (2) become

$$
\mathbf{V}_{i}=\mathbf{V}+\mathbf{\Omega} \times \mathbf{r}
$$

It is important to notice that if an object on the rotating system has $\mathbf{V}=\mathbf{0}$, even then it will have an associated velocity due to rotation: $\mathbf{V}_{i}=\boldsymbol{\Omega} \times \mathbf{r}$ (Fig. $1)$.
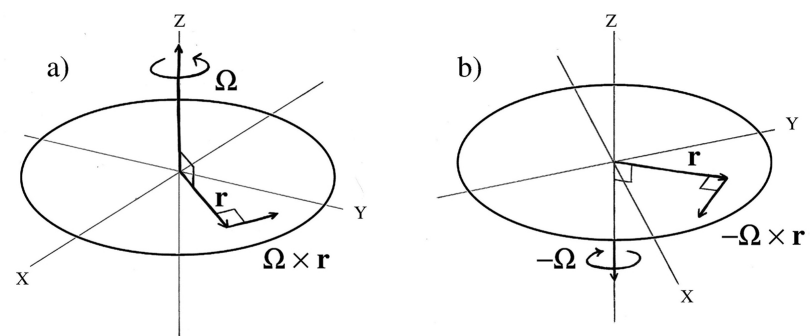

Figure 1: Change of the position vector $(\mathbf{r})$ in the inertial reference system $(d \mathbf{r} / d \mathrm{t})_{i}$ without movement in the noninertial reference frame $(d \mathbf{r} / d \mathbf{t}=\mathbf{0})$, a) When movement of the plate is clockwise and b) When movement of the plate is counterclockwise.

Similarly, the acceleration in the inertial frame is given by

$$
\left(\frac{d \mathbf{V}_{i}}{d t}\right)_{i}=\frac{d \mathbf{V}_{i}}{d t}+\mathbf{\Omega} \times \mathbf{V}_{i}
$$

Substituting $\mathbf{V}_{i}$ on the right side of Eq. (4) from (3), to incorporate the apparent velocity in the inertial frame, we obtain

$$
\begin{array}{r}
\left(\frac{d \mathbf{V}_{i}}{d t}\right)_{i}=\frac{d \mathbf{V}}{d t}+\left(\boldsymbol{\Omega} \times \frac{d \mathbf{r}}{d t}\right)+ \\
\left(\mathbf{r} \times \frac{d \boldsymbol{\Omega}}{d t}\right)+(\boldsymbol{\Omega} \times \mathbf{V})+\boldsymbol{\Omega} \times(\boldsymbol{\Omega} \times \mathbf{r}) .
\end{array}
$$

And assuming constant angular velocity, the Eq. (5) is reduced to

$$
\left(\frac{d \mathbf{V}}{d t}\right)_{i}=\frac{d \mathbf{V}}{d t}+2 \boldsymbol{\Omega} \times \mathbf{V}+\boldsymbol{\Omega} \times(\boldsymbol{\Omega} \times \mathbf{r}) .
$$

When the sum of forces $(\mathbf{F})$ is considered on the rotating system (non-inertial), Newton's Law or momentum equation can be written as

$$
\frac{d \mathbf{V}}{d t}=\sum_{i} \frac{\mathbf{F}}{m}-2 \boldsymbol{\Omega} \times \mathbf{V}-\boldsymbol{\Omega} \times(\boldsymbol{\Omega} \times \mathbf{r}),
$$

where $-2 \boldsymbol{\Omega} \times \mathbf{V}$ and $-\boldsymbol{\Omega} \times(\boldsymbol{\Omega} \times \mathbf{r})$ are Coriolis acceleration and centrifuge acceleration respectively. Equation (7) includes other forces due pressure gradient, gravitation and friction, that we do not discuss in this paper.

Then, Coriolis acceleration can be expressed as Coriolis force per mass unit $(m)$ as 


$$
\frac{\mathbf{F}_{\text {Coriolis }}}{m}=-2 \boldsymbol{\Omega} \times \mathbf{V},
$$

which it is useful when it is difficult to quantify the mass, like in the case of moving fluids.

There are different derivations to the previous one, which has been made in order to not introduce terms like "fixed" and "rotating" system [9], or from a geometric perspective [10]. Even so, we consider the exposed theory in this paper is sufficient to achieve the goal.

\subsection{Aid of Angular Momentum Conservation}

It is possible to make a qualitative description of Coriolis force predicting its direction. We can do this by using angular momentum conservation.

Preliminarily, with the previous definitions of position vector $(\mathbf{r})$ and velocity vector $\left(\mathbf{V}_{i}\right)$, we can define the absolute angular momentum $(\mathbf{L})$ as

$$
\mathbf{L}=\mathbf{r} \times \mathbf{p}=\mathbf{r} \times m \mathbf{V}_{i},
$$

where $\mathbf{p}$ is the linear momentum. Moreover, if we assume constant mass, Eq. (9) can be written as

$$
\mathbf{L}=m\left(\mathbf{r} \times \mathbf{V}_{i}\right),
$$

Using Eq. (3) to get a new equation in terms of velocity on the rotating system (non-inertial), then the angular momentum per mass unit will be

$$
\frac{\mathbf{L}}{m}=\mathbf{r} \times \mathbf{V}+\mathbf{r} \times(\boldsymbol{\Omega} \times \mathbf{r}) .
$$

From Eq. (9) it follows that angular momentum is conserved only in the absence of torque $(\boldsymbol{\tau})$, i.e.

$$
\text { if } \quad \boldsymbol{\tau}=\mathbf{r} \times \frac{d \mathbf{p}}{d t}=\mathbf{0} \quad \Rightarrow \quad \frac{d \mathbf{L}}{d t}=\mathbf{0} .
$$

\section{Coriolis force direction using Angular Momentum Conservation and comparison with its direct calculation (qualitative vs. quantitative)}

In the qualitative description of Coriolis force direction, we study two cases: 3.1 ) Two-dimensional motion and 3.2) Three-dimensional motion.
We suppose instantaneous velocity movements, hence there is no torque, and angular momentum is conserved. Friction is neglected. In every case we refer to movements of a punctual object which has constant mass $(m)$.

\subsection{Two-dimensional motion}

The movements of a punctual object on a flat plate rotating with constant angular velocity $(\boldsymbol{\Omega})$ can be classified into three types: a) Radial, in which movement is made over a radius of the plate; b) Tangential, movement is perpendicular to the radius in which the object is located at that instant, and c) A combination of the preceding (Fig. 2).

The object moves with instantaneous velocity $\mathbf{V}$ $=(u, v, 0)$, that is, the considered movements occurs on XY plane.

\subsection{1. a) Radial movements}

In this case, movements are made over any radius $(\mathbf{r})$ and given that $\mathbf{r}$ and $\mathbf{V}$ are parallel then $\mathbf{r} \times$ $\mathbf{V}$ is zero. Thus by Eq. (11) we obtain only the second term $\mathbf{r} \times(\boldsymbol{\Omega} \times \mathbf{r})$ for the angular momentum per mass unit. Finally, in order to conserve angular momentum, a move toward a larger (smaller) radius should reduce (increase) the tangential velocity.

Let's use this qualitative fact about the direction of the force in the next example: take a flat plate rotating with angular velocity $\boldsymbol{\Omega}=(0,0, \Omega)$, let $\mathbf{V}$ $=(u, 0,0)$ be the velocity of the object, as shown in Fig. 3a. Observe that the object is moving from a radius $r_{1}$ toward larger one $r_{2}$, so that, to conserve the angular momentum there must be a force $(\mathbf{f})$ to

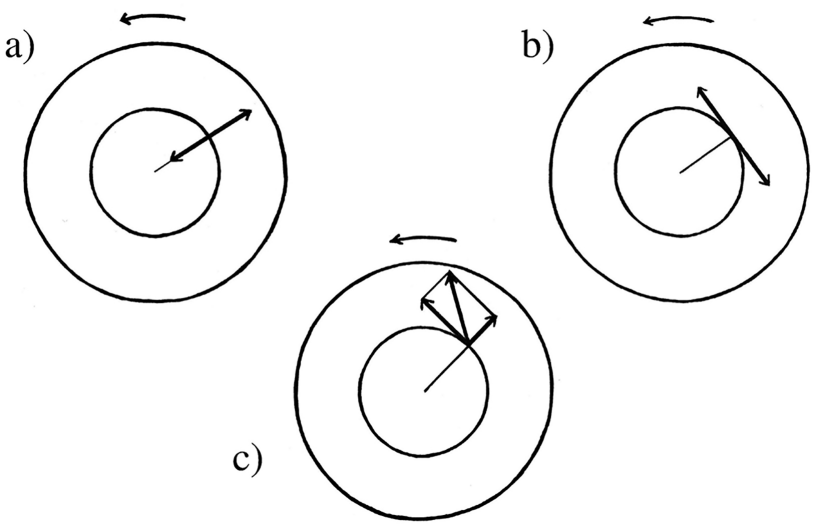

Figure 2: Movements on a rotating flat plate: a) radial, b) tangential and c) linear combination of previous both. 

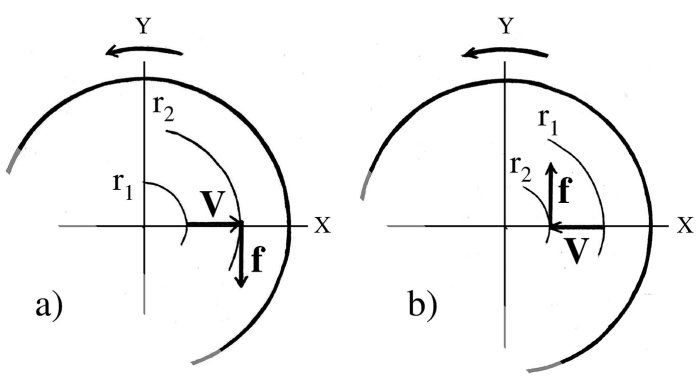

Figure 3: Radial movements $(\mathbf{V})$ on a rotating flat plate toward: a) a larger radius and b) a smaller radius. And its forces (f).

reduce the tangential velocity $\boldsymbol{\Omega} \times \mathbf{r}_{2}=\left(0, \Omega r_{2}, 0\right)$, and therefore $\mathbf{f}$ should have an opposite direction to it. Then, the force will have the vector form $(0,-f$, 0 ), where the negative sign represents that the force is going the opposite way to tangential velocity.

Now, we want to compare the direction of the force obtained qualitatively with the analytical calculation of the Coriolis force, with $\Omega=(0,0, \Omega)$ and $\mathbf{V}=(u, 0,0)$. By Eq. (8) we obtain

$$
\begin{array}{r}
\frac{\mathbf{F}_{\text {Coriolis }}}{m}=-2 \boldsymbol{\Omega} \\
\times \mathbf{V}=-2\left|\begin{array}{ccc}
\mathbf{i} & \mathbf{j} & \mathbf{k} \\
0 & 0 & \Omega \\
u & 0 & 0
\end{array}\right|= \\
-2 u \Omega \mathbf{j}=(0,-2 u \Omega, 0),
\end{array}
$$

which is fully consistent with the predicted direction of the force by using angular momentum conservation.

Let us consider a move toward a smaller radius (Fig. 3b) with $\mathbf{V}=(-u, 0,0)$ and keeping $\boldsymbol{\Omega}=(0,0$, $\Omega)$ as above. Since the object is going to a smaller radius, there must be a force to increase the tangential velocity in order to conserve angular momentum, then it will have the same direction of the tangential velocity $\boldsymbol{\Omega} \times \mathbf{r}_{1}$ and therefore the same sign, i.e., it will have the form $(0, f, 0)$. Calculating analytically Coriolis force as in Eq. (13) we have

$$
\frac{\mathbf{F}_{\text {Coriolis }}}{m}=2 u \Omega \mathbf{j}=(0,2 u \Omega, 0),
$$

which it is also in accordance with the predicted.

\subsection{2. b) Tangential Movements}

In this case, the velocity of the object $\mathbf{V}$ and the tangential velocity $\boldsymbol{\Omega} \times \mathbf{r}$ are parallels, so they can easily be added. So $\mathbf{V}$ can be expressed as $\boldsymbol{\Omega} \times \mathbf{r}_{n}$, where $r_{n}$ is a radius, which is a multiple of its current radius, i.e., $r_{n}=\alpha r_{1}$ where $\alpha$ is a scalar.

Suppose the case shown in Fig. 4a, where the object has a velocity $\mathbf{V}=(0, v, 0)=\boldsymbol{\Omega} \times \alpha \mathbf{r}_{1}$, then the equation of angular momentum per mass unit takes the form

$$
\begin{aligned}
\frac{\mathbf{L}}{m}= & \mathbf{r}_{1} \times\left(\mathbf{V}+\boldsymbol{\Omega} \times \mathbf{r}_{1}\right)=\mathbf{r}_{1} \times\left(\boldsymbol{\Omega} \times \alpha \mathbf{r}_{1}\right)+ \\
& \mathbf{r}_{1} \times\left(\boldsymbol{\Omega} \times \mathbf{r}_{1}\right)=(1+\alpha) \mathbf{r}_{1} \times\left(\boldsymbol{\Omega} \times \mathbf{r}_{1}\right),(15
\end{aligned}
$$

$\mathbf{V}$ is $\alpha$ times the tangential velocity due to rotation in that point. If $\alpha$ is positive, $\mathbf{V}$ has the same instantaneous direction as the rotation.

There is a circle of radius $r_{2}>r_{1}$ such that angular momentum per mass unit is the same as (15). Let $r_{2}=r_{1} \sqrt{ }(1+\alpha)$ and $\mathbf{V}=\mathbf{0}$, then the angular momentum per mass unit due to rotation $\boldsymbol{\Omega} \times \mathbf{r}_{2}$ is

$$
\begin{gathered}
\frac{\mathbf{L}}{m}=\mathbf{r}_{2} \times\left(\mathbf{0}+\boldsymbol{\Omega} \times \mathbf{r}_{2}\right)=\sqrt{1+\alpha} \mathbf{r}_{1} \times \\
\left(\boldsymbol{\Omega} \times \sqrt{1+\alpha} \mathbf{r}_{1}\right)=(1+\alpha) \mathbf{r}_{1} \times\left(\boldsymbol{\Omega} \times \mathbf{r}_{1}\right) .
\end{gathered}
$$

So, for angular momentum conservation, when tangential velocity increases there must be a force which impels the object toward a larger radius, having the form $(f, 0,0)$.
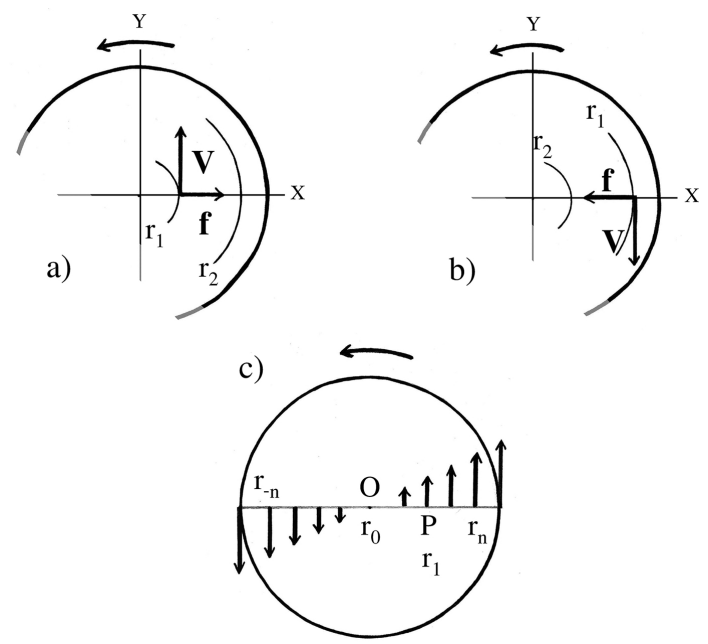

Figure 4: Tangential movements $(\mathbf{V})$ on a rotating flat plate, where: a) the tangential velocity increases and b) the tangential velocity decreases; and its forces (f). c) Tangential velocity due to rotation $(\Omega \times \mathbf{r})$ depending on the radius $r_{n}$. If the object is in a point $\mathrm{P}$ on the plate, the point $\mathrm{P}$ and the center $\mathrm{O}$ of the plate define a directed line segment OP. Radii are considered positive if they have the same direction of the OP segment, and negative if they have opposite direction. 
Calculating analytically the Coriolis force for $\mathbf{V}=$ $(0, v, 0)$, we have

$$
\frac{\mathbf{F}_{\text {Coriolis }}}{m}=2 v \Omega \mathbf{i}=(2 v \Omega, 0,0) .
$$

Again, this result is in agreement with the predicted one.

When $\mathbf{V}$ has the opposite direction than the rotation, reduces tangential velocity that corresponds to the radius in which the object is located (Fig. 4b), then the force impels the object toward a smaller radius $r_{2}$ as define above. This case occurs when $\alpha<0$ in Eq. (16). We need to clarify what is meant by "smaller radius". Consider the diameter which passes through the point where the object is located. We can define positive and negative radii associated to a point as directed line segments. If $\mathrm{O}$ is the center of the plate and $\mathrm{P}$ the current location of the object, then the segment OP defines a positive direction. Then a positive radius is one having the same direction as OP, and a radius with an opposite direction is a negative radius. So, if $-1 \leqslant \alpha \leqslant 0$, $r_{2}$ is a positive radius between the center of the plate and $r_{1}$, and if $\alpha<-1, r_{2}$ is a negative radius. In both cases $r_{2}$ is a smaller radius than $r_{1}$. To smaller radii correspond smaller tangential velocities due the rotation and conversely to larger radii correspond larger tangential velocities due to rotation, as shown in Fig. 4c.

\subsection{3. c) Linear combination of previous movements}

If a movement is a linear combination of previous ones, we can imagine that solution is also a linear combination of their solutions.

As $\mathbf{V}$ has the form $(u, v, 0)$, as shown in Fig. 5, the object is moving toward a larger radius and is increasing the tangential velocity. So in order to conserve the angular momentum, the force should reduce tangential velocity and should impel the object to a larger radius, having the form $\mathbf{f}=\left(f_{x}\right.$, $\left.-f_{y}, 0\right)$.

Calculating analytically the Coriolis force we have,

$$
\begin{aligned}
& \frac{\mathbf{F}_{\text {Coriolis }}}{m}=2 v \Omega \mathbf{i}-2 u \Omega \mathbf{j} \\
& =(2 v \Omega,-2 u \Omega, 0),
\end{aligned}
$$

which is according with the prediction.

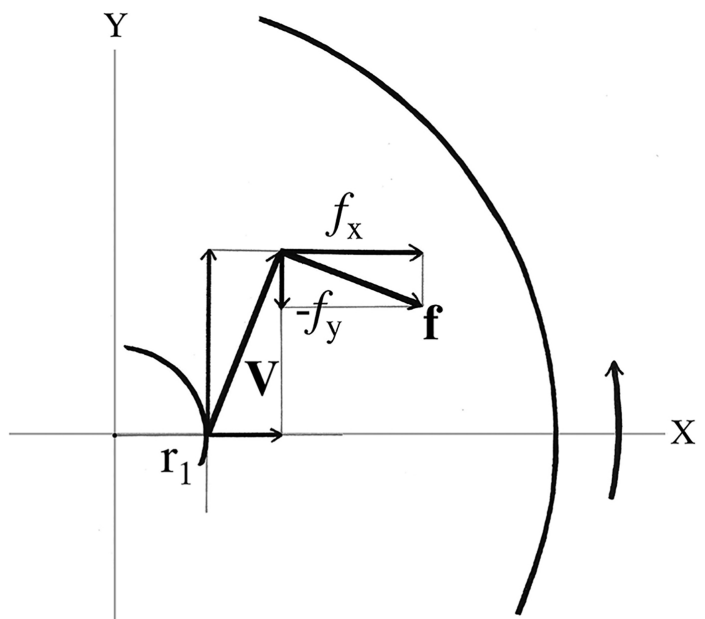

Figure 5: Movements (V) on a rotating flat plate, as linear combination of radial and tangential movements. And its forces (f).

\subsection{Three-dimensional motion}

Let us consider movements on a rotating solid sphere. In every point on the sphere, using Cartesian coordinates, it can be define a plane tangent to the surface, where the $\mathrm{X}$ axis coincides with a parallel, the $\mathrm{Y}$ axis coincides with a meridian and the $\mathrm{Z}$ axis is orthogonal to the plane.

Movements are considered again with instantaneous velocity. As in section 2.1 we denote $\mathbf{V}=d \boldsymbol{r} / d t$ $=(d x / d t, d y / d t, d z / d t)=(u, v, w)$, where movements are linear combination of $u, v$ and $w$ (Fig. $6)$.

It may be advantageous to name the poles as north $(\mathrm{N})$ and south $(\mathrm{S})$ and consequently name east and west, such that movements from east to west (west to east) correspond to speed $u$, from

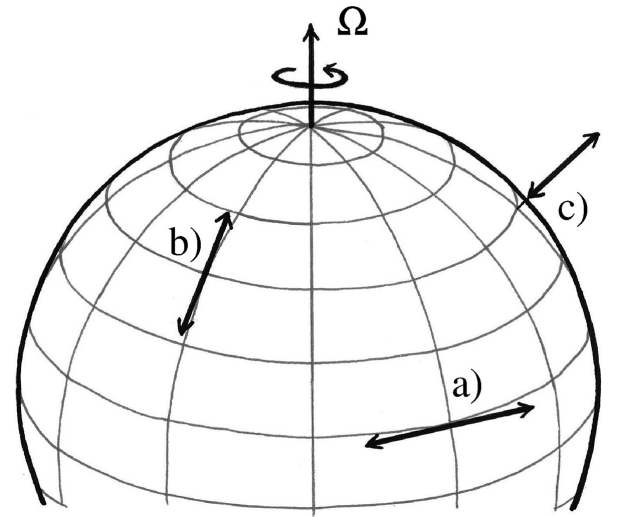

Figure 6: Movements on a rotating solid sphere, a) over a parallel, b) over a meridian and c) vertical movements.

Revista Brasileira de Ensino de Física, vol. 38, nº 3, e3304, 2016 
north to south (south-north) to speed $v$ and from up to down (down-up) to speed $w$. In the literature of atmospheric sciences, these three movements are known as: zonal, meridional and vertical movements respectively.

On the sphere, the angular velocity vector $(\boldsymbol{\Omega})$ depends on the angle known as latitude $(\phi)$. Moreover, $\boldsymbol{\Omega}$ has the same magnitude but different direction on each hemisphere (Fig. 7), where to each hemisphere correspond similar cases as shown ones on Fig. 1a and 1b. From Fig. 8 it can be deduced that angular velocity vector can be expressed as

$$
\boldsymbol{\Omega}=\left(0, \Omega_{y}, \Omega_{z}\right)=(0, \Omega \cos \phi, \Omega \sin \phi) .
$$

From Eq. (8) we can obtain an expression in Cartesian coordinates for $-2 \boldsymbol{\Omega} \times \mathbf{V}$, such that

$$
\begin{array}{r}
\frac{\mathbf{F}_{\text {Coriolis }}}{m}=(2 v \Omega \sin \phi-2 w \Omega \cos \phi) \mathbf{i}- \\
2 u \Omega \sin \phi \mathbf{j}+2 u \Omega \cos \phi \mathbf{k}
\end{array}
$$

Now we can analyze some interesting examples:

Let be a point between North Pole and the Equator, and let be a movement towards the East with $\mathbf{V}=(u, 0,0)$, this case is similar to the one studied on section 3.1.1. in which the tangential velocity is increasing, then there must be a force (f) which impels the object toward a larger radius. But, in the a)

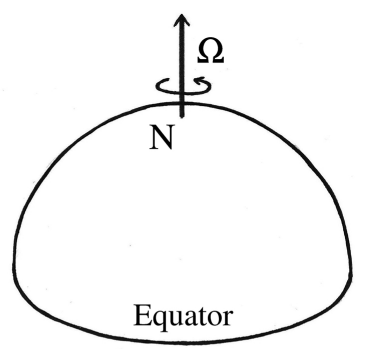

b)

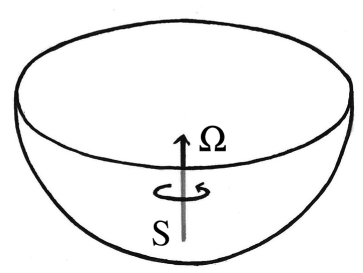

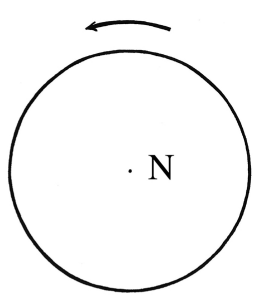

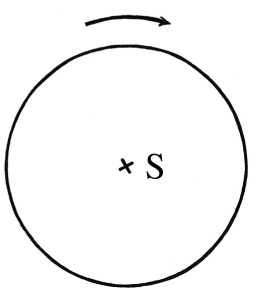

Figure 7: Oblique view of a rotating solid sphere: a) cut for North hemisphere (N), and top view, b) cut for South hemisphere (S), and bottom view. On the top and bottom views the dot indicates that the vector $\Omega$ comes out the surface and the cross indicates that vector $\Omega$ gets in the surface.

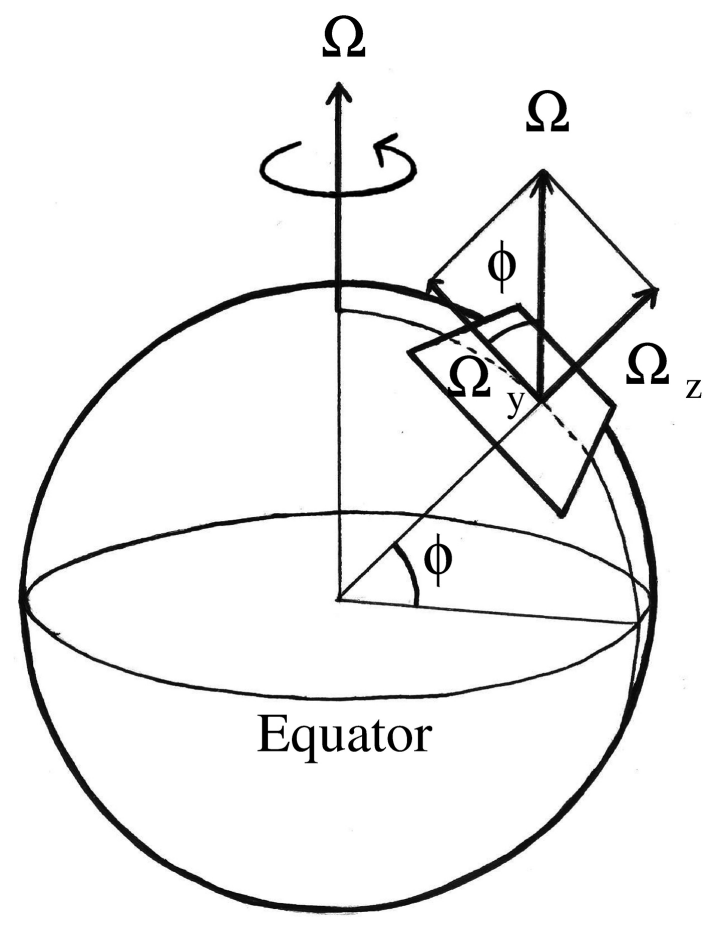

Figure 8: Oblique view of a rotating solid sphere, outlining the components of the vector $\Omega$ with respect the latitude angle $\phi$.

spherical case there are two possibilities for moving toward a larger radius: i) Moving toward the Equator and ii) Moving upward (Fig. 9a); so the force should have the form $\mathbf{f}=\left(0,-f_{y}, f_{z}\right)$. Substituting V on Eq. (20) we have

$$
\frac{\mathbf{F}_{\text {Coriolis }}}{m}=-2 u \Omega \sin \phi \mathbf{j}+2 u \Omega \cos \phi \mathbf{k}
$$

which coincides with expectations.

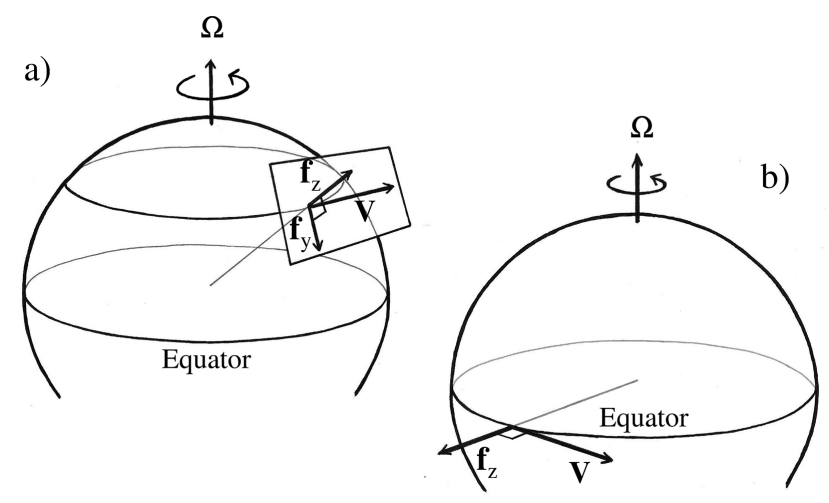

Figure 9: Movements (V) over a parallel on a rotating solid sphere and its forces (f) for: a) a latitude between Equator and North Pole $\left(0^{\circ}<\phi<90^{\circ}\right)$ and $\left.\mathrm{b}\right)$ on Equator $\left(\phi=0^{\circ}\right)$. 
Changing the previous example a bit, we obtain another interesting result. Let be the same condition as before $\mathbf{V}=(u, 0,0)$, but now at the Equator of the sphere. Likewise as in the previous case, there must be a force (f) which impels the object toward a larger radius, but the movement can not be over a meridian because the object in any case would move to a smaller radius, then the only possibility to go to a larger radius is to go upward (Fig. 9b). So the force should have the form: $\mathbf{f}=\left(0,0, f_{z}\right)$. Calculating Coriolis force analytically with Eq. (20) and taking $\phi=0^{\circ}$ on Equator we obtain

$$
\frac{\mathbf{F}_{\text {Coriolis }}}{m}=2 u \Omega \mathbf{k}=(0,0,2 u \Omega)
$$

as we wanted.

\section{Conclusions}

The angular momentum conservation allows us to predict the direction of Coriolis force of a moving object on a rotating flat plate or rotating sphere (with constant angular velocity). If the object moves toward a larger (smaller) radius, in order to conserve angular momentum, there must be a force to reduce (increase) the tangential velocity. If the object moves parallel to tangential velocity, for angular momentum conservation, when tangential velocity increases (decreases) there must be a force which impels the object toward a larger (smaller) radius.

Many of the theories of physics created in the nineteenth century are complicated for students because their final form, represented by equations, has concentrated a number of concepts into an explanation which becomes dense, difficult to follow, and hard to understand. It is better for didactic reasons, to analyze the ideas implied by those fundamental concepts. The explanation could be longer, but allows student to build simpler steps to go thru. The simple term $-2 \boldsymbol{\Omega} \times \mathbf{V}$ involves too many information, it resumes all said in this paper without being obvious all the implications that we have discussed.

The issue is valid in different levels and its relationship with other disciplines take increasing relevance [11-15].

\section{Acknowledgments}

The authors are grateful to Mary Lynne Evans and Ernesto Caetano for reviewing the text and style correction.

\section{References}

[1] G. Coriolis, L'Académie Des Sciences XIII, 268 (1831).

[2] G. Coriolis, L'Académie Des Sciences XVII, 142 (1835).

[3] H.G. Close and P.R.L. Heron, Am. J. Phys. 79 (10), 1068 (2011).

[4] N.A. Phillips, Bull. Amer. Meteor. Soc. 81, 299 (2000).

[5] A. Persson, Bull. Amer. Meteor. Soc. 79, 1373 (1998).

[6] A.G. Schmidt, Am. J. Phys. 54, 755 (1986).

[7] M. Alonso and E.J. Finn, University Physics Vol. I, Mechanics (Addison-Wesley, Reading, 1967).

[8] F. Viniegra-Heberlein, Mecánica, Libro I (Universidad Nacional Autónoma de México, México, 2007).

[9] G. Vandegrift, Am. J. Phys. 63, 663 (1995).

[10] D.H. McIntyre, Am. J. Phys. 68, 1097 (2000).

[11] O.A. Haugland, Phys. Teach. 47, 546 (2009).

[12] A. Tandon and J. Marshall, Phys. Teach 48, 292 (2010).

[13] L. Dallen and D.E. Neuenschwander, Am. J. Phys. 79, 326 (2011).

[14] D.A. Manjarres, W.J. Herrera and R.A. Díaz, Am. J. Phys. 81, 597 (2013).

[15] G.N. Mill, V.S. da Costa and A. de M. Paiva, Revista Brasileira de Ensino de Física 37, 4302 (2015). 